\title{
Error analysis in circuits building at the quantum computing platform IBM Quantum Experience
}

\author{
E. O. Samsonov \\ ITMO University, Kronverkskiy, 49, St. Petersburg, 197101, Russia \\ Edi.samsonov@gmail.com
}

PACS 03.65.Yz, 03.67.-a

DOI 10.17586/2220-8054-2017-8-2-272-276

\begin{abstract}
There are many quantum computing systems, some of which are still being developed today. To develop quantum calculation systems, IBM provides access to the 5-qubit quantum computer 'IBM Quantum Experience'. Quantum computers must deal with the loss of information due to environmental disturbances. Quantum systems cannot be completely isolated. Noise can be a cause of different errors in the quantum circuits. In this work, we observe distortions in quantum circuits and investigate the noise stability of different quantum gates. We investigate a method for calculating the quantum state of the superconducting qubit, used in 'IBM Quantum Experience', after an interaction with a quantum operator.
\end{abstract}

Keywords: quantum computing, quantum information, IBM Quantum Experience, distortions of quantum circuits.

Received: 14 January 2017

Revised: 18 February 2017

\section{Introduction}

Quantum systems have interactions with the environment. These interactions are presented as noise in quantum computing systems. The loss of information due to environmental disturbances is termed decoherence [1]. We need to develop an understanding of these noise processes so that they may be better controlled.

The evolution of the quantum system is considered in [2,3]. For different types of quantum computing systems, there are several ways to increase the system stability with respect to the effect of noise. Interactions between superconducting quantum systems, used in 'IBM Quantum Experience', and the most significant sources of noise, are investigated in [4-7]. The effect of noise in quantum circuits is considered in [8-10]. The errors may result in distortions of quantum algorithms. Research on the Grover's algorithm stability with respect to perturbations of quantum logic circuit elements is represented in [11]. Quantum computers use quantum error correction to protect information from decoherence errors and other quantum noise. Quantum error correction is considered in [8,10].

In this work, we use an open access to the 'IBM Quantum Experience' computer [12], to investigate distortions of quantum circuits. This gives us the opportunity to observe known quantum circuits with respect to noise. Various circuits can be used for implementation of the same quantum gate. We consider different circuits for implementation of the same well-known quantum single-qubit and two-qubit gates, such as NOT and CNOT. Also, we investigate the NOT gate and two types of controlled NOT gate, such as CNOT and 'Toffoli' gates with respect to noise stability.

A superconducting qubit called 'transmon' is used in 'IBM Quantum Experience' [4]. We investigate a method for calculating the quantum state of this superconducting qubit after interaction with the quantum operator, which includes a source of noise. Thus, we can calculate the quantum state without using the quantum computer and investigate the qubit with respect to noise stability.

\section{Quantum circuits}

A graphical user interface for programming a quantum processor in 'IBM Quantum Experience' is 'Quantum Composer' [12]. There are single-qubit gates in 'Quantum Composer', such as Pauli operators and Clifford operators. Also, it contains a two-qubit gate CNOT. We will consider different circuits for implementation of the same $\mathrm{X}$ gate, which is equivalent to a classical NOT, and different circuits for implementation of the same CNOT gate.

The single-qubit $\mathbf{X}$ gate is a Pauli operator, which represents bit-flip. The $\mathrm{X}$ gate is represented in the library of 'Quantum Composer'. If the initial state is $|0\rangle$, after an interaction with the $\mathrm{X}$ gate, the probability of the expected state $|1\rangle$ is 0.976 . We can observe that there are some errors, which are due to noise processes in the circuit. 
We can create a circuit which works like the $\mathbf{X}$ gate. $\mathbf{X}=\mathbf{H Z H}$, where $\mathbf{Z}$ - Pauli operator, $\mathbf{H}-$ Hadamard gate. If the initial state is $|0\rangle$, after an interaction with this circuit for implementation of the $\mathrm{X}$ gate, the probability of the expected state $|1\rangle$ is 0.934 .

Two different circuits for implementation of the same quantum gate with respect to the noise stability can be compared. We notice that the quantum circuit for implementation of the $\mathbf{X}$ gate is worse with respect to the noise stability than the $\mathbf{X}$ gate which is represented in the library of 'Quantum Composer'.

The two-qubit CNOT gate functions as an exclusive OR gate in conventional digital logic. If the initial state is $|00\rangle$, after an interaction with the CNOT gate, the probability of the expected state $|00\rangle$ is 0.913 . In this experiment, there are more decay channels, and there is the interaction between two qubits.

We can create a circuit for a controlled-V operation if we can find three circuits $\mathbf{A}, \mathbf{B}, \mathbf{C}$, such that $\mathbf{A B C}=\mathbf{I}$ and $\mathrm{e}^{i a} \mathbf{A X B X C}=\mathbf{V}$ [13]. We have added a control qubit to $\mathbf{X}$ gate to implement a controlled-X operation, which is an equivalent to a CNOT. For the controlled-X operation $\mathrm{e}^{i a} \mathbf{A X B X C}=\mathbf{X}$, where $a=\pi / 2$, the circuits $\mathbf{A}, \mathbf{B}, \mathbf{C}$, is given by the Pauli and Clifford gates: $\mathbf{A}=\mathbf{H Y}, \mathbf{B}=\mathbf{H X T}, \mathbf{C}=\mathbf{T}$. Combining these circuits as shown in [13], we will obtain the controlled-X gate (Fig. 1).

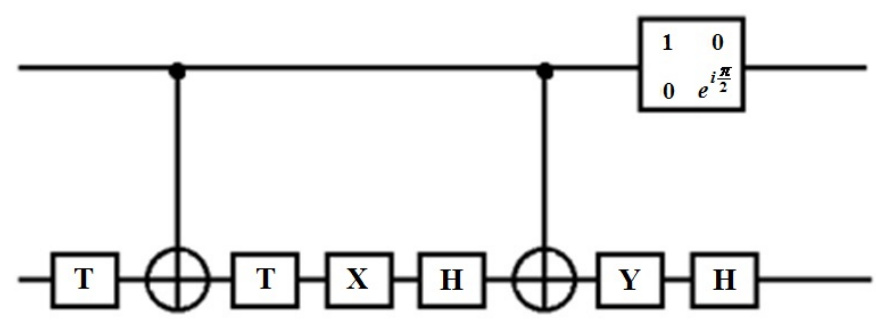

FIG. 1. Controlled-X operation

If the initial state is $|00\rangle$, after an interaction with this controlled-X gate, the probability of the expected state $|00\rangle$ is 0.901 .

After this experiment, we compare different circuits for implementation of the same quantum gate with respect to the noise stability. The quantum circuit for implementation of the CNOT gate, which was created, is worse with respect to the noise stability than the CNOT gate, which is represented in the library of 'Quantum Composer'. But we can see that the values from the second experiment approximately equal the values from the first experiment.

Also, it is interesting to investigate the NOT gate and two types of controlled NOT gates, such as CNOT and Toffoli, with respect to the noise stability.

The set of allowed connections is defined by the schematic of the device. We cannot use the known three-qubit CCNOT gate, however, we can use another circuit for implementation of the quantum three-qubit gate CCNOT. It is important to investigate this quantum circuit with respect to the noise stability. The CCNOT gate is illustrated in the Fig. $2[8,13]$.

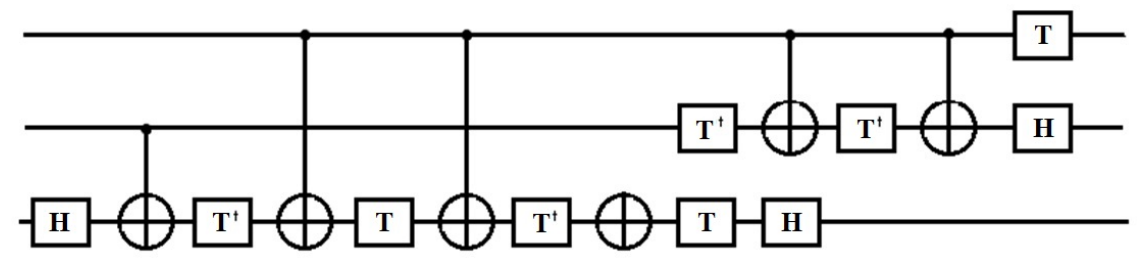

FIG. 2. Circuit for implementation of the quantum three-qubit CCNOT gate

If the initial state is $|00\rangle$, after the interaction with the circuit for implementation of the quantum three-qubit gate CCNOT gate, the expected state $|00\rangle$ is 0.746 . The probability of the expected state after the $\mathbf{X}$ gate is 0.976 , and the probability of the expected state after the CNOT gate is 0.913 . We can notice a significant decrease in the noise stability of the quantum circuit for implementation of the quantum three-qubit gate.

\section{Interaction with an external field}

A promising type of a quantum bit is the Josephson-junctions based quantum bit. There are three types of these qubits: phase [5]; flux [6]; and charge [5,7,14]. These types of qubits have a great scalability, advanced 
capabilities for managing states, relatively high coherence time. Such macroscopic quantum systems have been described previously [5]. The impact of the most significant sources of noise is considered in [4-7]. A new type of superconducting qubit was introduced in [4]. They proposed a superconducting qubit called a 'transmon'. The 'transmon' is closely related to the 'Cooper pair box' qubit; it has a superconducting 'island' with two Josephson junctions (with Josephson energy $\mathrm{E}_{j}$, which can be controlled by an external magnetic field) and gates capacitance, which control the charge energy $\mathrm{E}_{c}$ for one Cooper pair. If there is only one or zero Cooper pair in the 'island', we can get a two-level system (qubit). The Hamiltonian for this system [4,5] is given by:

$$
\mathbf{H}_{0}=-\frac{1}{2} B_{z} \boldsymbol{\sigma}_{z}-\frac{1}{2} B_{x} \boldsymbol{\sigma}_{x}
$$

where $B_{z}$ - is determined by the charging energy $E_{c}, B_{x}$ - is determined by the Josephson energy $E_{j}$ and the magnetic field through a superconducting ring. $\boldsymbol{\sigma}_{z}$ and $\boldsymbol{\sigma}_{x}$ are the Pauli operators. The 'transmon' is designed to operate in a regime of significantly increased ratio of Josephson energy to the charging energy of $E_{j} / E_{c}$. Its charge dispersion decreases exponentially with the $E_{j} / E_{c}$. Analysis of the full system in [4] shows that this benefit is not compromised by the increased noise in other channels. This type of qubit is used in IBM Quantum Experience.

It is interesting to investigate the dynamics of a superconducting qubit proposed in [4]. We present a method for calculating the quantum state of this superconducting qubit, after interaction with the quantum operator which includes a source of noise.

We will observe the interaction between the qubit and the external field. The impact of the external field can change the qubit state. Firstly we consider the ideal unitary evolution, which is represented by the logical operator NOT.

Evolution of the quantum system with time is given by:

$$
i \hbar \frac{\partial|\phi(t)\rangle}{\partial t}=\mathbf{H}_{0}|\phi(t)\rangle \text {. }
$$

If we integrate this equation, we get:

$$
|\phi(t)\rangle=\mathrm{e}^{-\frac{i}{h} t \mathbf{H}_{0}}|\phi(t)\rangle .
$$

Here, the $\mathbf{H}_{0}$, Hamiltonian of the system with a spin $1 / 2$ is given by (1).

Then the operator of the evolution is given by:

$$
\mathrm{e}^{\frac{-i}{\hbar} t \mathbf{H}_{0}}=\exp \left[\frac{i}{2 \hbar} t\left(B_{z} \boldsymbol{\sigma}_{z}+B_{x} \boldsymbol{\sigma}_{x}\right)\right]=\mathbf{E} .
$$

If only the external field $B_{x}$ is applied to the system, $\mathbf{E}$ will be given by:

$$
\mathbf{E}(\psi)=\exp \left[\frac{i t B_{x} \boldsymbol{\sigma}_{x}}{2 \hbar}\right]=\left(\begin{array}{cc}
\cos \frac{\psi}{2} & i \sin \frac{\psi}{2} \\
i \sin \frac{\psi}{2} & \cos \frac{\psi}{2}
\end{array}\right),
$$

where $\psi=B_{x} t / \hbar$.

In other words, we can obtain a unitary evolution. For certain $B_{x}$ and $t$ values, we can get the operator $\mathbf{X}$ accurate to a phase factor.

There is some noise in real quantum systems. Control pulses create unwanted external fluctuations. In contact with its surroundings, the coherence decays with time in a process called quantum decoherence. The time evolution of a system with noise can be described with the master equations [8]. Secondly, we consider the interaction between the system and a control pulse with a Gaussian noise as a reversible process [2]. In work [15], the minimum energy path was calculated with Gaussian process regression for transitions such as spin rearrangements.

We will consider evolution of system, which is represented by the logical operator NOT with some noise. We write Hamiltonian of the system $\mathbf{H}(t)$ as a sum $\mathbf{H}_{0}$ and the component $\boldsymbol{\sigma}_{x} V(t)$, which describes the timedependent noise field V(t) [5]. For the system, which is observed, the noise field can be created by the externally applied flux [2]:

Evolution of the system is given by:

$$
\mathbf{H}(t)=\mathbf{H}_{0}+\boldsymbol{\sigma}_{x} V(t)
$$

$$
|\phi(t)\rangle=\mathrm{e}^{-\frac{i}{\hbar} t \mathbf{H}_{0}}\left|\phi(t)_{I}\right\rangle,
$$

$\mathrm{e}^{-\frac{i}{\hbar} t \mathbf{H}_{0}}=\mathbf{E}(\psi)$, where $\psi=1.57$. Thus, this evolution operator, accurate to the phase factor, equals the Pauli operator $\mathbf{X}$. Thus, $\left|\phi(t)_{I}\right\rangle$ is the state vector in the interaction picture, and $\left|\phi(t)_{I}\right\rangle$ is given by:

$$
\left|\phi(t)_{I}\right\rangle=\mathrm{e}^{\frac{i}{\hbar} t \mathbf{H}_{0}} V(t)|\phi(0)\rangle
$$


where $|\phi(0)\rangle=\left|\phi(0)_{I}\right\rangle$,

where $\mathbf{U}(t)_{I}=\mathrm{e}^{\frac{i}{\hbar} t \mathbf{H}_{0}} \mathbf{U}(t)$.

$$
\left|\phi(t)_{I}\right\rangle=\mathbf{U}(t)_{I}\left|\phi(0)_{I}\right\rangle
$$

Then:

$$
\mathbf{U}(t)=\mathrm{e}^{-\frac{i}{\hbar} t \mathbf{H}_{0}} \mathbf{U}(t)_{I} .
$$

This quantum operator describes the system with a unitary evolution operator, which has been described, and a time-dependent noise field.

From the equation for the motion of the operator $\mathbf{U}(t)$, we obtain:

Then:

$$
i \hbar \frac{\partial \mathbf{U}(t)}{\partial t}=\left(\mathbf{H}_{0}+\boldsymbol{\sigma}_{x} V(t)\right) \mathrm{e}^{-\frac{i}{\hbar} t \mathbf{H}_{0}} \mathbf{U}(t)_{I} .
$$

$$
i \hbar \frac{\partial \mathbf{U}(t)_{I}}{\partial t}=\mathbf{V}(t)_{I} \mathbf{U}(t)_{I}
$$

where $\mathbf{V}(t)_{I}=\sigma_{x} V(t)$. If we integrate this equation, we will get an accurate, to the second order, small parameter $\varepsilon=\frac{1}{\hbar} \int_{0}^{t} V(\tau) d \tau$ :

$$
\mathbf{U}(t)_{I}=\mathbf{I}-\frac{i}{\hbar} \boldsymbol{\sigma}_{x} \int_{0}^{t} V(\tau) d \tau+\frac{1}{2}\left(-\frac{i}{\hbar} \boldsymbol{\sigma}_{x} \int_{0}^{t} V(\tau) d \tau\right)^{2} .
$$

Then, $\mathbf{U}(t)$ is given by:

$$
\mathbf{U}(t)=i \boldsymbol{\sigma}_{x}+\frac{1}{\hbar} \mathbf{I} \int_{0}^{t} V(\tau) d \tau-\frac{i}{\hbar^{2} 2} \boldsymbol{\sigma}_{x}\left(\int_{0}^{t} V(\tau) d \tau\right)^{2} .
$$

We consider $V(\tau)$ as a Gaussian process [5], $\mathbf{U}(t)$ will be random, the expected value of the variable part in (14) is the integral of the expected value of $V(\tau)$. Typical parameters of the system: the flux field magnitude is $\approx 10^{-6} \Phi_{0}$, where $\Phi_{0}$ is the magnetic quantum flux [2,6], and the interaction time $t$ is 1 ns [2]. Thusly, we derive the evolution operator:

$$
\mathbf{U}=\left(\begin{array}{ll}
0.196 & 0.981 \\
0.981 & 0.196
\end{array}\right)
$$

We have obtained the operator NOT with a bit flip channel. There are errors in a circuit because of the interaction with an external field.

If the initial state is $|0\rangle$, we get:

$$
\mathbf{U}|0\rangle=a|0\rangle+b|1\rangle
$$

where $a=0.196, b=0.981$. The probability of the state $|0\rangle$ is $|a|^{2}=0.038$, the probability of the state $|1\rangle$ is $|b|^{2}=0.962$. We have calculated the quantum state of this superconducting qubit, after the interaction with the quantum operator, which includes a source of noise. These values are approximately equal to the values from the experiment, which we have got from the quantum computing platform. If the initial state is $|0\rangle$, after an interaction with the $\mathbf{X}$ gate, the probability of the state $|0\rangle$ is 0.024 , and the probability of the expected state $|1\rangle$ is 0.976 . So, we have calculated the quantum state without using the quantum computer and investigate the qubit with respect to the noise stability.

\section{Acknowledgments}

This work was partially financially supported by the Government of the Russian Federation (grant 074U01).

\section{References}

[1] Zurek W.H. Decoherence, einselection, and the quantum origins of the classical. Rev. Mod. Phys., 2003, 75, P. $715-775$.

[2] Blum K. Density matrix theory and applications. Plenum press, New York, 1981, 242 pp.

[3] Gardiner C. Stochastic methods for natural sciences. Springer Science, New York, 2004, 538 pp.

[4] Koch J., Yu T.M., Gambetta J., Houck A. A., Schuster D. I., Majer J., Blais A., Devoret M. H., Girvin S. M., Schoelkopf R.J. Charge-insensitive qubit design derived from the Cooper pair box. Phys. Rev. A, 2007, 76, P. 042319/1-19.

[5] Makhlin Yu. Quantum coherence in mesoscopic superconducting systems and quantum computing. Doctor dissertation, Landau Institute for Theoretical Physics, Moscow, 2004.

[6] Yoshihara F., Harrabi K., Niskanen A.O., Nakamura Y., Tsai J.S. Decoherence of flux qubits due to 1/f flux noise. Phys. Rev., 2006, 97, P. 167001. 
[7] Averin D.V., Ruggiero B., Silvestrini P. Macroscopic Quantum Coherence and Quantum Computing. Springer Science and Business Media, New York, 2001, 460 pp.

[8] Nielsen M.A., Chuang I.L. Quantum Computation and Quantum Information. Cambridge University Press, Cambridge, 2001,670 pp.

[9] Aharonov D., Kitaev A., Nisan N. Quantum circuits with mixed states. Proceedings of 'The thirtieth annual ACM symposium on Theory of computing'. Dallas, May 24-26, ACM New York, 1998, P. 20-30.

[10] Kitaev A.Yu. Quantum computations: algorithms and error correction. Uspekhi Mat. Nauk, 1997, 52(5), P. 53-112.

[11] Gubaidullina K.V., Chivilikhin S.A. Theoretical research of the distortion of quantum circuit in Grover's algorithm. Journal of Physics. Conference Series, 2016, 735, P. 012074-1-012074-6.

[12] Retrieved from https://quantumexperience.ng.bluemix.net/qstage/\#/community.

[13] Barenco A., Bennett C.H., Cleve R., DiVincenzo D.P., Margolus N., Shor P., Sleator T., Smolin J.A., Weinfurter H. Elementary gates for quantum computation. Phys. Rev. A, 1995, 52, P. 3457-3467.

[14] Makhlin Y., Shnirman A. Dephasing of Solid-State Qubits at Optimal Points. Phys. Rev., 2004, 92, P. 178301.

[15] Koistinen O-P., Maras E., Vehtari A., J'onsson H. Minimum energy path calculations with Gaussian process regression. Nanosystems: Physics, Chemistry, Mathematics. 2016, 7(6), P. 925-935. 